\title{
A Location-Aware Routing Metric (ALARM) for Multi-Hop, Multi-Channel Wireless Mesh Networks
}

\author{
Eiman Alotaibi, Sumit Roy \\ Dept. of Electrical Engineering \\ U. Washington Box 352500 \\ Seattle, WA 98195 \\ eman76,roy@ee.washington.edu
}

\begin{abstract}
The importance of suitable link-aware routing metrics for multi-hop (mesh) wireless networks is well-established. Channel-to-link assignments impact the distribution of cochannel links and contribute to inter-flow and intra-flow interference. In this paper we present A new Location-Aware Routing Metric (ALARM) that better captures the resulting co-channel interference as a function of channel assignments. The link metrics used are sensitive to the location of nearby co-channel links that are within interference or carrier sensing range; these can be pre-calculated and used with any routing algorithm. The performance of ALARM is compared with Weighted Cumulative Expected Transmission Time metric (WCETT) via OPNET over a comprehensive range of network parameters for linear and 2-D grid topologies.
\end{abstract}

\section{INTRODUCTION}

The relevance of cross-layer approaches to wireless network design is, by now, well-accepted. These methods typically attempt to optimize a suitable metric at the network layer while incorporating lower-layer (link and multiple access) parameters. In this work, we focus on the important problem of selecting the appropriate metrics select the route in wireless multi-hop networks, which differs significantly from that in wired networks, due to the broadcast nature of wireless [1]. Hence, modeling of interference that is strongly influenced by link and multiple access (layers 1-2) functions is key towards improving path selection at the network layer. The advent of improved link aware metrics for routing [3], [4], [6] has naturally evolved into joint channel assignment and route selection formulations in recognition of their respective contributions to the task of interference management across layers 1-3. Good link-aware metrics should possess a few general characteristics: they should capture the interaction between co-channel links along the same flow ('self or intraflow interference') and those across different flows ('cross or inter-flow interference') in the network.

All proposed link-aware metrics such as these in [2], [4], [5] and [6] are based on heuristic improvements to the traditional routing formulation. The latter, devised for wired networks, has a sum-of-link cost structure, where the link costs are determined based on link bandwidth, data rate, etc. The goal has been to incorporate the above 'inter-flow' and 'intraflow' interference components into the standard shortest-path formulation, that has typically been done on a heuristic basis. We note that nearly all proposed metrics use the Expected Transmission Time (ETT) as a basis for link cost. For instance, the focus of [6] is the transmission interference that is integrated with Expected Transmission Time (ETT), whereas [5] adds the switching cost to the total path cost of $\left(\sum E T T_{i}\right)$.

In this work we focus on the Weighted Cumulative Expected Transmission Time metric (WCETT) [4] since it is the source from which we derive our new metric (ALARM). WCETT was proposed in as an enhancement to Expected Transmission Count (ETX) [3] that uses only the loss rate but not link bandwidth. WCETT is a weighted average of two components: the first is the usual sum of transmission times along the path while the second seeks to add diversity to the channel allocation along a flow by encouraging better use of all available channels 
(and thus mitigating 'inter-flow' interference). However, the diversity measure is based on $\sum_{i \in p} E T T_{i}$ over all the cochannel links in a flow irrespective of the location of the links, which is a significant weakness. From simulation evidence, WCETT is known to perform well for shorter paths but its performance degrades when the path length increases. Clearly including the impact of interference range on any Wireless Mesh Network (WMN) routing metric can be expected to yield performance improvements, as has already been suggested in [9] and [10].

\section{A Location-Aware Routing Metric (ALARM)}

\section{A. ALARM description}

Our new metric consists of two components: the first is identical to that of WCETT. However, the second component represents our effort to enhance the WCETT performance by adding a location-dependent co-channel component that seeks to include the impact of distance between the co-channel links, i.e.,

$$
A L A R M=(1-\alpha) \sum_{i \in p} E T T_{i}+\alpha \sum_{i \in p}\left\{N_{i} \sum_{j \in S_{i}} w_{i j}\right\}
$$

where ETT is the Expected Transmission Time given by

$$
E T T=E T X \times \frac{P S}{B}
$$

The Expected Transmission Count (ETX) was introduced in [3], and PS and B refer to packet size and link bandwidth respectively. The weights $w_{i j}$ are determined based on cochannel links $i, j$ that share the same channel as shown in Fig. 1, as expressed by the following:

$$
w_{i j}=\left\{\begin{array}{cc}
\frac{1}{2 R_{I}} & d_{i j}<R_{c s} \\
\frac{1}{d_{i j}} & R_{c s}<d_{i j}<R_{I} \\
0 & R_{I}<d_{i j}
\end{array}\right.
$$

Note that the above presumes a static link-to-channel assignment, assuming all co-channel links transmitting simultaneously.

- $\mathrm{p}$ is the set of all links along a path;

- $S_{i}$ is a set which includes all the links within carrier sensing or interference range of $\operatorname{lin} k_{i}$;

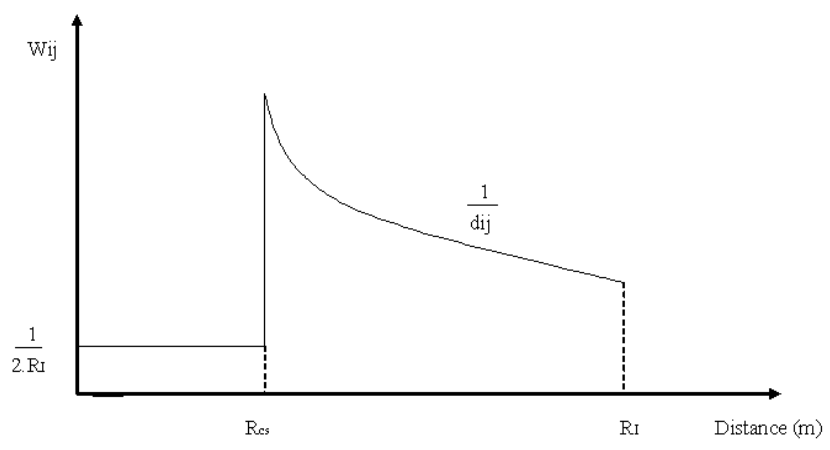

Fig. 1. The weight $w_{i j}$ vs. distance.

- $N_{i}$ is the number of active co-channel links of $l i n k_{i}$, in other words, $N_{i}$ is the size of set $S_{i}$;

- $R_{I}$ is the Interference range; it is the maximum distance at which $\operatorname{link}_{i}$ can be interfered by any other co-channel links;

- $R_{c s}$ is the carrier sensing range;

- $d_{i j}$ is the distance between the receiver of $l i n k_{i}$ and the sender of the co-channel $\operatorname{link}_{j}$;

As in [4], the first term in Eq. (1) captures the end-to-end path latency based on the sum of individual link delays. The second term represents the impact of bottleneck link in the path and is the sum of the pairwise co-channel links weights $\left(w_{i j}\right)$ which we called the location factor. The importance of the location factor is highlighted when the number of active links increases within the carrier-sensing and interference ranges. We divide the co-channel links into two sets based on their location (distance) from the reference link. The co-channel links that are located within $R_{c s}$ are in set $C S_{i}\{\}$ while set $I_{i}\{\}$ includes the co-channel links that are out of $R_{c s}$ but still within $R_{I}$ of the current link. The union of the sets $I_{i}\{\}$ and $C S_{i}\{\}$ creates

$$
S_{i}\{\}=I_{i}\{\} \cup C S_{i}\{\}
$$

The current link $\left(\operatorname{link}_{i}\right)$ refers to the link on which we focus at a given instant. The critical ranges such as carrier-sensing range $\left(R_{c s}\right)$ and Interference range $\left(R_{I}\right)$ are specifically defined in [7].

The value of $w_{i j}$ depends on the mutual distance $d_{i j}$ between co-channel $l i n k_{i}$ and $l i n k_{j}$ relative to the critical 
This full text paper was peer reviewed at the direction of IEEE Communications Society subject matter experts for publication in the WCNC 2008 proceedings.

ranges $R_{c s}$ and $R_{I}$. In this paper we assume that $R_{c s}<R_{I}$ since the opposite case eliminates the existence of $R_{I}$ which means that we only deal with $R_{C S}$.

When $d_{i j}<R_{c s}$, $\operatorname{link} k_{j} \in C S_{i}$, which implies that the senders of $l i n k_{i}$ and $l i n k_{j}$ are within mutual carrier-sense range. Within this range, the effect of the co-channel link is unrelated to its specific location, i.e., the throughput of the current link will be degraded by the same factor in case of any co-channel link within $R_{c s}$ of the reference; therefore the value of $w_{i j}$ must be constant for all co-channel links in $R_{c s}$. Also, the weight of $w_{i j}$ assigned must be smaller than its value when there is interference between both links. This is so because, if the senders are within carrier-sense range, it assures that at least one flow is active whereas, with interfering flows, the overall loss in data rate over both links is greater. So, we assign $w_{i j}$ a value equal to $\left(2 R_{I}\right)^{-1}$ since $R_{I}$ is the maximum distance counted in ALARM and weight is inversely proportional to this distance, i.e., $\left(2 R_{I}\right)^{-1}$ is the smallest value that can be used as a cost. One more advantage of using $R_{I}$ in this part is connection between the data rate and the interference range from one side and the throughput from the other side. So, whenever the data rate changes, metric weight will compromise to match this change. Therefore, the values of $w_{i j}$ in both sets $\left(I_{i}\right.$ and $\left.C S_{i}\right)$ are comparable.

On the other hand, the second value of $w_{i j}$ when $R_{c s}<$ $d_{i j}<R_{I}$ varies with the location of the co-channel links. The longer the distance, the less is the interference between the two links as measured by the distance between the receiver of $l i n k_{i}$ and the sender of $l i n k_{j}$. Therefore, the value of $w_{i j}$ decreases with increasing $d_{i j}$ until it drops to zero when $d_{i j}$ exceeds $R_{I}$. It is important here to note that the value of $w_{i j} \neq w_{j i}$, in other words, $w_{i j}$ and $w_{j i}$ are not symmetric because of the different distances between the sender and the receiver of each pair.

Finally, $w_{i j}=0$ if the separation between the pair of cochannel links exceeds the interference range. This property improves the overall spatial reuse because any co-channel link outside the critical range of $\operatorname{lin} k_{i}$ is not counted in ALARM; therefore, that channel can be reused beyond the interference range of $l i n k_{i}$ with zero effect on that particular link. Furthermore, the value of $N_{i}$ confirms that the selected path is the best path by assuming that the number of overall co-channel links along the selected path is the minimal.

We next provide two different examples showing computation of the location-dependent factor: $\left(\sum_{i \in p}\left\{N_{i} \sum_{j \in S_{i}} w_{i j}\right\}\right)$.

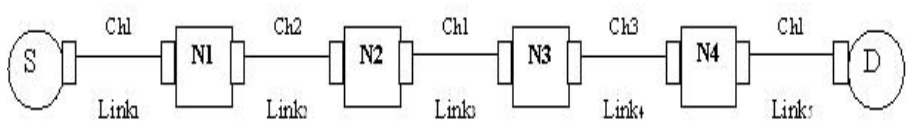

Fig. 2. Three interfering co-channel links.

First, in Figure 2, three co-channel links are sharing a channel (ch1). The steps to calculate the location factor are as follow:

- The path includes 5 links, which means $\mathrm{i}=1,2,3,4$ and 5 in the outer summation.

- Starting with the first link, $\operatorname{link}_{1}$, $(\mathrm{i}=1)$ : there are two co-channel links $\left(\operatorname{link}_{3}\right.$ and $\left.\operatorname{link}_{5}\right) . \operatorname{Link}_{3}$ is within interference range of $\operatorname{lin}_{1}$ while $\operatorname{lin}_{5}$ is located just out of the interference range of $\operatorname{link}_{1}, S_{1}=\left\{\operatorname{lin} k_{3}\right\}, \mathrm{j}=1$. $d_{11}=10 \mathrm{~m}$, and $w_{11}=\frac{1}{10}$. Since there is only one link in set $S_{1}$, then $N_{1}=1$.

- For $\operatorname{link}_{2}(\mathrm{i}=2)$ : there is no co-channel links. Thus, $S_{2}=\emptyset, N_{2}=0, w_{2 j}=0$.

- For $\operatorname{link}_{3}(\mathrm{i}=3)$ : there are again two co-channel links $\left(\operatorname{link}_{1}\right.$ and $\left.l i n k_{5}\right)$; one is within interference range $\left(\operatorname{link}_{5}\right)$ and one is out of range $\left(\operatorname{lin} k_{1}\right) . S_{3}=$ $\left\{\operatorname{link}_{5}\right\}, N_{3}=1, \mathrm{j}=1 . d_{31}=10 \mathrm{~m}, w_{31}=\frac{1}{10}$.

- For $\operatorname{link}_{4}(\mathrm{i}=4)$ : there is no co-channel links. Thus, $S_{4}=\emptyset, N_{4}=0, w_{4 j}=0$.

- For $\operatorname{link}_{5}(\mathrm{i}=5)$ : there are two co-channel link $\left(\operatorname{lin} k_{1}, \operatorname{link}_{3}\right)$ but none is within interference range of $\operatorname{link}_{5} \cdot S_{5}=\emptyset, N_{5}=0$.

- For Fig. $2 \sum_{i=1}^{5}\left\{N_{i} \sum_{j=1}^{1} w_{i j}\right\}=1 *\left(\frac{1}{10}\right)+0 *(0)+$ $1 *\left(\frac{1}{10}\right)+0 *(0)+0 *(0)$.

In the second example in Figure 3, there are two links sharing a channel (ch1). The following steps are used to calculate the location factor:

- The path includes 5 links, $\mathrm{i}=1,2,3,4$ and 5 in the outer 


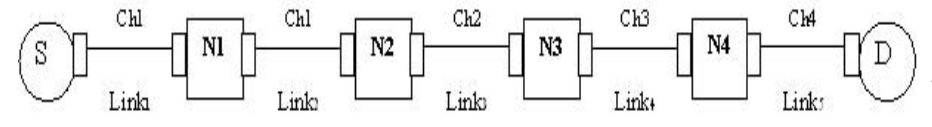

Fig. 3. Two co-channel links within carrier-sense range.

summation.

- First link $\operatorname{link}_{1}$, (i=1): there is one co-channel link $\left(S_{1}\right.$ $=\left\{\operatorname{link}_{2}\right\}$ ) within carrier-sensing range of $\operatorname{lin} k_{1} \mathrm{j}=1$. $d_{\max }=30 \mathrm{~m}$, and $w_{11}=\frac{1}{2 * 30}, N_{1}=1$.

- For $\operatorname{link}_{2}$, $(\mathrm{i}=2)$ : same as the first link. $S_{2}=\left\{\operatorname{link}_{1}\right\}$, $\mathrm{j}=1 . d_{\max }=30 \mathrm{~m}$, and $w_{21}=\frac{1}{2 * 30}, N_{1}=1$.

- For links $\operatorname{link}_{3}, \operatorname{link}_{4}$ and $\operatorname{link}_{5}(\mathrm{i}=3,4,5)$ : there are no co-channel links. Thus, $S_{3}=S_{4}=S_{5}=\emptyset, N_{3}=$ $N_{4}=N_{5}=0, w_{i j}=0$.

- For Fig. $3 \sum_{i=1}^{5}\left\{N_{i} \sum_{j=1}^{1} w_{i j}\right\}=1 *\left(\frac{1}{2 * 30}\right)+1 *\left(\frac{1}{2 * 30}\right)+$ $0 *(0)+0 *(0)+0 *(0)$.

\section{B. ALARM vs. WCETT: Comparison}

The Weighted Cumulative Expected Transmission Time (WCETT) [4] is based on the Expected Transmission Count (ETX) modified to include the link bandwidth as follows:

$$
W C E T T=(1-\beta) \sum_{i=1}^{n} E T T_{i}+\beta \max _{1 \leq j \leq k} X_{j}
$$

Here, $k$ is the number of channels, $n$ is the number of hops, and ETT is defined in Eq. (2). $X_{j}$ is the sum of (expected) transmission times over all hops on a given channel $\mathrm{j}$, i.e.,

$$
X_{j}=\sum_{\text {hopi on channelj }}^{n} E T T_{i} 1 \leq j \leq k
$$

WCETT has two components: the first represents the sum of expected transmission times along all hops on a path. The second term reflects the subset of hops that will have the most impact on the throughput along the path due to link congestion. The use of $0 \leq \beta \leq 1$ leads to a weighted average of the linear sum-of-link costs (first) term with the new non-linear component that seeks to penalize the subset of links with higher costs. From simulation evidence, WCETT performs well for shorter paths but its performance drops when the path length increases.
Since both ALARM and WCETT have identical first term, the key difference lies in the construction of the second term which in both cases intends to promote channel diversity. ALARM supports channel spatial reuse by a weighting scheme accounts for co-channel interference within a maximum distance of $R_{I}$. Because the ALARM value of each link given by Eq. (6) can be pre-calculated and stored, it is possible to implement any routing algorithm that can select the path with the smallest sum (the best path), i.e.,

$$
\operatorname{ALARM}_{i}=(1-\alpha) E T T_{i}+\alpha N_{i} * \sum_{j \in S_{i}} w_{i j}
$$

\section{EVALUATION}

In this section, we evaluate the performance of ALARM and compare it with WCETT for three different topologies: chain networks, two parallel chains and 2-D Wireless Mesh Networks (WMN). All the simulation examples contain a bottleneck link in each case which affects the overall endto-end throughput. The impact of this bottleneck link on throughput naturally depends on the location of the co-channel links.

All simulations are done within OPNET version 11.0. The traffic is generated by layer-3 (IP-layer) flows which are used to collect the end-to-end throughput. In this work, we modify the OPNET source code to calculate the ETT directly by OPNET at the WLAN module. ETT (expected value of transmission time) on each link is measured during the simulation by adding a time stamp to each packet. At the end of the simulation, all the transmission times are averaged to get the final value of 1-hop ETT for each link. Without loss of generality, following [4], the values of $\alpha$ and $\beta$ in ALARM and WCETT respectively are equivalent $(\alpha=\beta=0.5)$ for all simulations.

\section{A. Single Chain Topology}

The first evaluation tests the chain (1-dimension) topology where we assign different channel assignments with parameters given in Table I. For this topology, we examined more than 100 different cases based on varying the channel assignment, link data rates, total number of channels, number of simultaneous paths, interference range or carrier-sensing range. After 
TABLE I

SINGLE CHAIN TOPOLOGY FOR SIMULATION.

\begin{tabular}{|c|c|}
\hline Path length & 5 hops. \\
\hline Interference range $\left(R_{I}\right)$ & $30 \mathrm{~m}$ (for $10 \mathrm{~m} 1$-hop distance) \\
& $59 \mathrm{~m}$ (for $20 \mathrm{~m}$ 1-hop distance) \\
\hline Carrier-Sensing Range $\left(R_{c s}\right)$ & $10 \mathrm{~m}, 20 \mathrm{~m}$ or $30 \mathrm{~m}$ \\
\hline Nodes separation distance $(\mathrm{d})$ & $10 \mathrm{~m}$ or $20 \mathrm{~m}$ \\
\hline Link data-rate & $12 \mathrm{Mbps}(=1000 \mathrm{pps})$ or \\
& $6 \mathrm{Mbps}(=500 \mathrm{pps})$ \\
\hline Packet size & 1500 bytes \\
\hline Transmission power & $1 \mathrm{~mW}$ \\
\hline
\end{tabular}

collecting the throughput for each case, we calculate the values of both ALARM and WCETT metric and ascertain whether ALARM or WCETT selected the path with higher throughput.

According to the simulation results, ALARM outperforms WCETT, i.e., WCETT selects the path with low throughput while ALARM usually selects the path with the maximum or very-close-to-the-maximum throughput. In the few cases where ALARM doesn't find the best path in the network, the difference doesn't exceed $4 \%$ from the optimum.

As shown in Figure 4, the new metric solves up to 14 wrong selections by WCETT. However, ALARM still fails to identify the optimum path in 6 cases (denoted by 'error') - three of these errors happened for the same channel assignment which means that the problem is caused by the ETT values and not due to the location-dependent part of ALARM metric. The other error occurred when comparing two different channel assignment but the difference in throughput here is negligible.

\section{B. Two Parallel Chains}

As a continuation of the first simulation experiments, we added a second path to the single chain topology. 22 different cases of two parallel chains topology are tested by locating two simultaneous paths separated by variable distances and assigning different channel assignment for each case. The simulation setup is listed in Table II. Figure 4 also shows the summary of simulation results - unlike WCETT, ALARM always selects the best path for all the 22 tested cases.

To confirm the quality of ALARM, more tests are applied for different data rates $(6,12,24$ and 48) Mbps with different interference ranges.
TABLE II

2-D TOPOLOGY SIMULATION SETUP

\begin{tabular}{|c|c|}
\hline Path length & 5 hops. \\
\hline Interference range $\left(R_{I}\right)$ & $30 \mathrm{~m}$ \\
\hline Carrier Sensing Range $\left(R_{c s}\right)$ & $10 \mathrm{~m}$ \\
\hline Nodes separation distance $(\mathrm{d})$ & $10 \mathrm{~m}$ \\
\hline Link data-rate & $12 \mathrm{Mbps}(=1000 \mathrm{pps})$ \\
\hline Packet size & 1500 bytes \\
\hline Transmission power & $1 \mathrm{~mW}$ \\
\hline
\end{tabular}

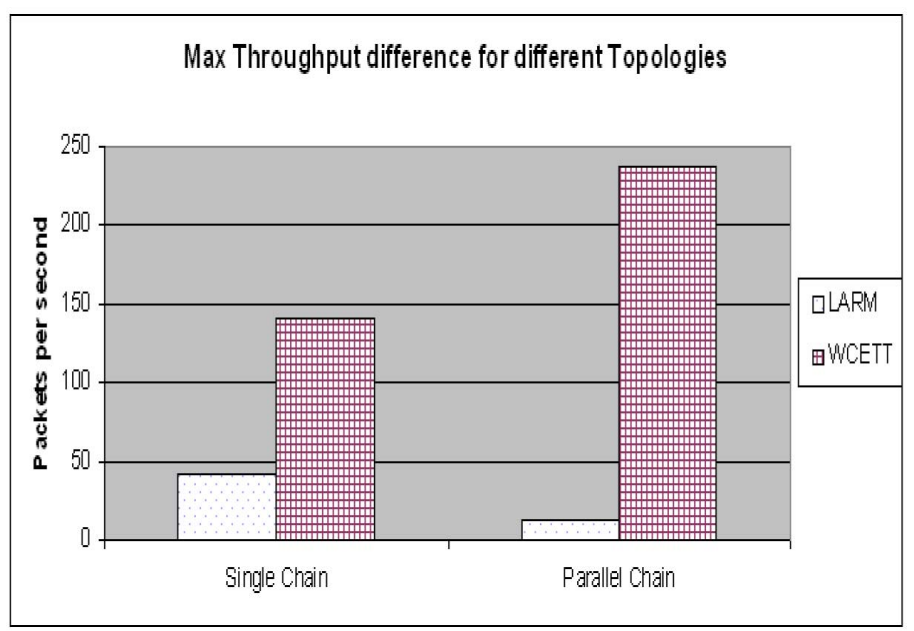

Fig. 4. Summary of ALARM and WCETT performance in Single and parallel chain topologies. WCETT is unable to select the best path in more than 35 cases (out of 100 cases) and ALARM in 8 cases. The difference in the throughput between the (wrongly) selected path from the optimum is used as a measure in the above.

\section{2-D Mesh}

In this section, we apply both WCETT and ALARM to the same $4 \times 4$-mesh network. First, we ran a single flow of 6-hop. Then, another 6-hop flow is added resulting in two simultaneous flows. Finally, four 4-hop flows are also added. ALARM records better results compared to WCETT as shown in Table IV (the same result is also clearly shown in Figure 6). Even when eight flows are running simultaneously, ALARM still gives higher throughput. Although ALARM was implemented based on a single path, it provides high throughput when it is used in a mesh network. This can be attributed to the fact that ALARM takes into consideration the effect of all surrounding co-channel links that could lead to loss in link throughput. It is also found that WCETT reuses the links which have low values of ETT in many paths regardless of the inter-flow interference links which become overloaded in time and create bottlenecks.

The channel assignment for this $4 \times 4$ mesh network was 
TABLE III

MESH TOPOLOGY SIMULATION SETUP

\begin{tabular}{|c|c|}
\hline number of nodes & 16 \\
\hline mesh size & $4 \mathrm{x} 4$ \\
\hline number of radios & 2 \\
\hline Total number of channels & 4 \\
\hline Interference range $\left(R_{I}\right)$ & $30 \mathrm{~m}$ \\
\hline Carrier Sensing Range $\left(R_{c s}\right)$ & $10 \mathrm{~m}$ \\
\hline Nodes separation distance $(\mathrm{d})$ & $10 \mathrm{~m}$ \\
\hline Link data-rate & $12 \mathrm{Mbps}(=1000 \mathrm{pps})$ \\
\hline Packet size & $1500 \mathrm{bytes}$ \\
\hline Transmission power & $1 \mathrm{~mW}$ \\
\hline
\end{tabular}

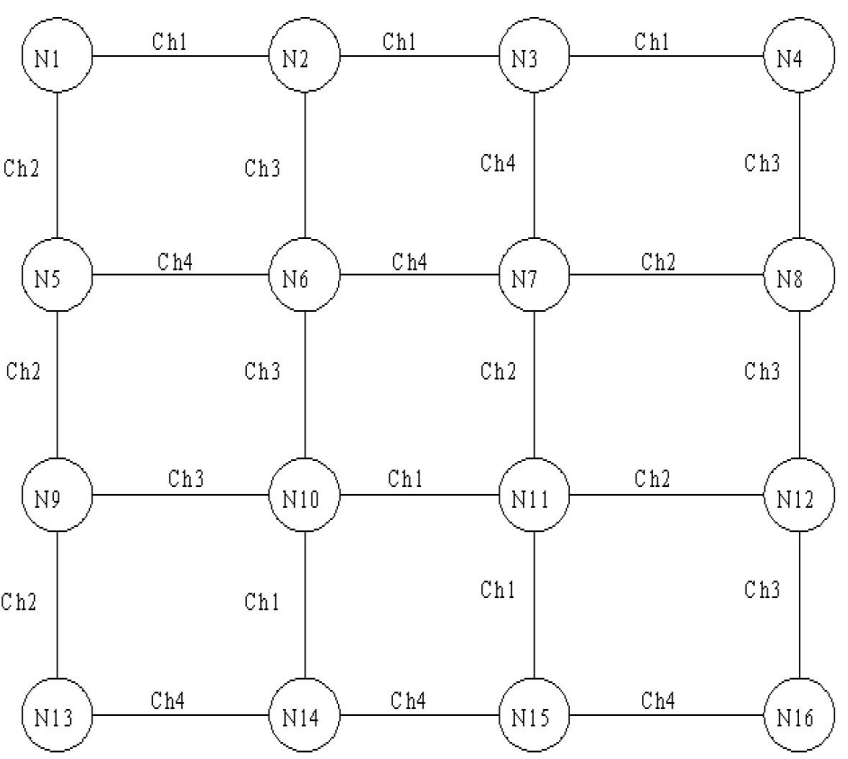

Fig. 5. $4 x 4$ mesh network setup and channel assignment

randomly selected as shown in Figure 5. Since there is no routing algorithm implemented here, all path-selection calculations were done manually, off-line.

\section{CONCLUSION}

In this work, we presented a new routing metric, ALARM, that uses knowledge of the locations of surrounding co-channel links to select the best path. Using OPNET simulation, it is shown that ALARM performs very well compared to WCETT.

TABLE IV

COMPARISON OF ALARM VS. WCETT THROUGHPUT (PACKETS/SECONDS) IN MESH TOPOLOGY

\begin{tabular}{|c|c|c|c|}
\hline Number of flows & description & ALARM & WCETT \\
\hline 1 & 5 hops & 28 & 19 \\
\hline 2 & 5 hops & 50 & 45 \\
\hline 4 & 4 hops & 200 & 170 \\
\hline 5 & 4 of 4 hops and 1 of 6 hops & 210 & 181 \\
\hline 6 & 4 of 4 hops and 2 of 6 hops & 250 & 236 \\
\hline 8 & 4 of 3 hops and 4 of 4 hops & 378 & 280 \\
\hline
\end{tabular}

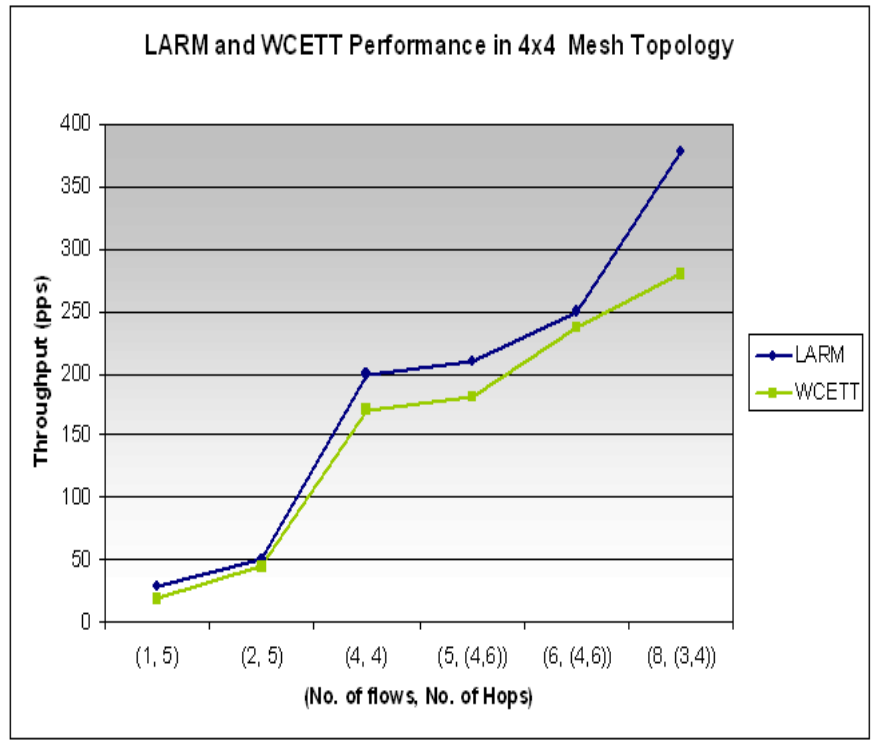

Fig. 6. Comparison of ALARM vs. WCETT throughput (packets/seconds) in Mesh Topology.

Although ALARM can be run using any on-demand routing algorithm like WCETT, our future work will focus on creating a new routing algorithm that matches the features of ALARM in order to yield the best expected performance.

\section{REFERENCES}

[1] D. De Couto, D. Aguayo, B. Chambers and R. Morris, "Performance of MultiHop Wireless Networks: Shortest Path is Not Enough," Proc. HotNets, ACM Sigcomm, 2002.

[2] Y. Yang, J. Wang and R. Kravets, "Designing routing metrics for mesh networks," 1st proc., IEEE workshop on wireless mesh network (WiMesh), 2005.

[3] D. De Couto et al., "High throughput Path Metric for multi-hop wireless networks," Proc., IEEE MOBICOM 2003.

[4] R. Draves et al., "Routing in Multi-Radio Multi-Hop Wireless Mesh Networks," Proc., MOBICOM 2004.

[5] P. Kyasanur et al., "Routing and Link-layer Protocols for Multi-channel Multi-Interference AD Hoc Wireless Networks," 2006.

[6] J. Park et al., "Expected Data Rate: An Accurate High-Throughput Path MEtric For Multi-Hop Wireless Routing," Proc., IEEE SECON, 2005.

[7] S. Roy, H. Ma, R. Vijaykumar and J. Zhu, "Optimizing 802.11 Wireless Mesh Networks Performance Using Physical Carrier Sensing," (submitted), 2006. [Technical report version available at https: \\www.ee.washington.edu \techsite $\backslash$ papers $\backslash$ documents $\backslash$ UWEETR-2006-0005.pdf

[8] H. Viswanathan et al., "Throughput-Range Tradeoff Wireless Mesh Backhaul Networks," Proc., IEEE Jornal on Comm. 2006.

[9] L. Iannone et al., "Cross-Layer Routing in Wireless Mesh Networks," 1st International Symposium in Wireless Communication Systems (ISWCS'04).

[10] L. Iannone et al., "Evaluating a cross-layer approach for routing in Wireless Mesh Networks," Telecommunication Systems Journal (Springer) Special issue: Next Generation Networks - Architectures, Protocols, Performance, vol. 31, No. 2-3, pg. 173-193, March 2006. 